\title{
Cephalometric craniofacial features of patients with Sagliker syndrome: a primary analysis of our experience
}

\author{
Jiao-Ping $\mathrm{Mi}^{1 \#}$, Peng $\mathrm{He}^{1 \#}$, Kai Shi ${ }^{2}$, Shao-Yan Feng ${ }^{1}$, Xian-Zhen Chen ${ }^{1}$, Qing-Qing $\mathrm{He}^{3}$, \\ Ming-Yue Zhao ${ }^{4}$, Ping-Jiang Ge ${ }^{5}$, Yun-Ping Fan ${ }^{1,6}$
}

${ }^{1}$ Department of Otolaryngology Head and Neck Surgery, The Fifth Affiliated Hospital of Sun Yat-sen University, Zhuhai, China; ${ }^{2}$ Department of Stomatology, The Fifth Affiliated Hospital of Sun Yat-sen University, Zhuhai, China; ${ }^{3}$ Department of Otolaryngology Head and Neck Surgery, Zhuhai Maternal and Child Health Hospital, Zhuhai, China; ${ }^{4}$ The Graduate School of Zunyi Medical University, Zunyi, China; ${ }^{5}$ Department of Otolaryngology Head and Neck Surgery, Guangdong Provincial General Hospital, Guangzhou, China; ${ }^{6}$ Department of Otolaryngology Head and Neck Surgery, The Seventh Affiliated Hospital of Sun Yat-sen University, Shenzhen, China

Contributions: (I) Conception and design: PJ Ge, YP Fan; (II) Administrative support: None; (III) Provision of study materials or patients: JP Mi, K Shi, YP Fan; (IV) Collection and assembly of data: JP Mi, P He, SY Feng, QQ He, MY Zhao; (V) Data analysis and interpretation: JP Mi, P He, XZ Chen; (VI) Manuscript writing: All authors; (VII) Final approval of manuscript: All authors.

\#These authors contributed equally to this article.

Correspondence to: Ping-Jiang Ge. Department of Otolaryngology Head and Neck Surgery, Guandong Provincial General Hospital, Guangzhou, China. Email: gepingjiang@aliyun.com; Yun-Ping Fan. Department of Otolaryngology Head and Neck Surgery, The Fifth Affiliated Hospital of Sun Yat-sen University, Zhuhai, China; Department of Otolaryngology Head and Neck Surgery, The Seventh Affiliated Hospital of Sun Yat-sen University, Shenzhen, China. Email: fanyp@mail.sysu.edu.cn.

Background: Sagliker syndrome (SS) is characterized by a severe uglifying facial appearance resulting from untreated or inadequately treated secondary hyperparathyroidism (SHPT). To date, the craniofacial morphology of patients with SS has yet to be analyzed. The present research sought to cephalometrically evaluate the craniofacial features of patients with SS and to perform an in-depth analysis of their serum biochemical parameters, with the aim of furthering the theoretical basis for the early diagnosis and prevention of SS.

Methods: A retrospective chart review of 9 patients who fulfilled the diagnostic criteria for SS were included in this study, and their serum biochemical parameters were collected. After subjecting standard lateral cephalometric radiographic images to correction for distortions caused by magnification followed by digitization, we conducted a cephalometric analysis. Student's two-tailed $t$ tests or Mann-Whitney U tests were used to analyze the data. Thirty-three patients with patients with SHPT alone were also included as controls.

Results: The lower anterior facial height (ANS-ME) and total anterior facial height (N-Me) measurements of patients with SS were significantly increased compared to those of the controls. The angles between the Frankfort horizontal, palatal, and occlusal planes and the mandibular plane, were greater in the SS group than in the control group, as was the gonial angle. Patients with SS also exhibited a significantly larger maxillary protrusion angle and relative position of the maxilla to the mandible. Most patients with SS had class II malocclusion, whereas most of the controls exhibited normal occlusion. Soft tissue largely followed the same pattern as craniofacial changes. Our investigation also showed that among patients with SHPT, female sex, longer duration of dialysis, and higher serum levels of alkaline phosphatase and intact parathyroid hormone were associated with development to SS.

Conclusions: Patients with SS show facial and biochemical differences compared to patients with SHPT. Female sex, long dialysis duration, and high serum levels of intact parathyroid hormone and alkaline phosphatase may be potential risk factors for SS.

Keywords: Craniofacial morphology; chronic renal failure; cephalometric analysis; sagliker syndrome; secondary hyperparathyroidism (SHPT)

Submitted Feb 23, 2021. Accepted for publication May 27, 2021. 
doi: 10.21037/atm-21-1544

View this article at: https://dx.doi.org/10.21037/atm-21-1544

\section{Introduction}

Sagliker syndrome (SS), first described by Sagliker in 2004 , is a rare entity occurring in patients with late-stage chronic kidney disease in the course of overt secondary hyperparathyroidism (SHPT). SS is characterized by a severe uglifying facial appearance resulting from extremely severe maxillary, mandibular, and nasal deformities, and dental anomalies, as well as pathologically benign softtissue oral neoplasms (Figure 1). It also entails numerous other manifestations including short stature, irregularly scattered skull changes, class II maxillary and mandibular malocclusion, fingertip changes, knee and scapula deformity, hearing loss, and neuropsychiatric disorders (1-3).

SS has received much research attention since its discovery in the early $21^{\text {st }}$ century. Cases of facial skeletal changed in patients with late and severe chronic kidney disease have been documented sporadically $(4,5)$; however, currently, there are no detailed cephalometric data available on the relationship between craniofacial abnormalities and SS. In the present research, we carried out a cephalometric evaluation of the craniofacial characteristics of patients with SS together with an in-depth analysis of their serum biochemical parameters, and then compared these parameters with those of patients with simple SHPT. We hope that our findings will further the theoretical basis for the early diagnosis and clinical prevention of SS. We present the following article in accordance with the STROBE reporting checklist (available at https://dx.doi. org/10.21037/atm-21-1544).

\section{Methods}

\section{Study participants}

A retrospective chart review was performed of all patients with SHPT who were treated at the Fifth Affiliated Hospital of Sun Yat-sen University from 2013 to 2020, and their cephalometric and serum biochemical parameters were collected. Nine patients with SS were enrolled including 3 males (age range, 42-49 years; mean age, 45.58 years) and 6 females (age range, 36-46 years; mean age, 41.32 years). Each participant was diagnosed with SS, the confirmation of which was based on the presence of the above-described clinical manifestations during long- term follow-up and 1 of the following criteria being met: a facial skeletal deformity affecting the individual's social activities; or a change in the individual's facial appearance judged by at least 3 family members or physicians. A control group comprising 33 patients with SHPT was also enrolled and included 18 males (age range, 32-56 years; mean age: 48.75 years) and 15 females (age range, $40-55$ years; mean age, 46.61 years). Table 1 displays the age and sex distributions in the SS and control groups. Among the enrolled patients, glomerulonephritis and idiopathic kidney disease were the most common etiologies of chronic renal failure (CRF). All participants had received renal dialysis for a minimum of 2 years. Patients with obvious disharmony of facial features, maxillary-mandibular discrepancy, or prior major orthodontic treatment were excluded from the control group. The study was approved by the Ethics Committee of the fifth affiliated hospital of Sun Yat-sen University. Participants gave their consent after being fully informed of the study's purpose. The study was conducted in accordance with the Declaration of Helsinki (as revised in 2013).

\section{Cephalometric evaluation}

For the evaluation of their skeletal and dental features, all participants underwent standard lateral cephalometric radiography, which was performed by the same radiographer adopting a standard technique in all cases. Radiographs were obtained at a constant distance with the patient positioned $2.2 \mathrm{~m}$ apart from the $\mathrm{X}$-ray source with their teeth in centric occlusion and the lips relaxed. To standardize the distance between the patient and the film, as well as X-ray exposure and magnification, participants' heads were kept in position with a cephalostat. Each radiograph was traced on 0.002" acetate paper by the same investigator. For the avoidance of possible bias, the radiographs were traced in a randomized order and soft-tissue profiles were excluded. Cephalometric landmark points are shown in Figure 2, and Figure 3 shows templates of cephalometric analysis for both SS and control groups.

\section{Laboratory measurements}

The serum levels of calcium (mmol/L), phosphorus $(\mathrm{mmol} / \mathrm{L})$, and alkaline phosphatase (ALP) (U/L) were 

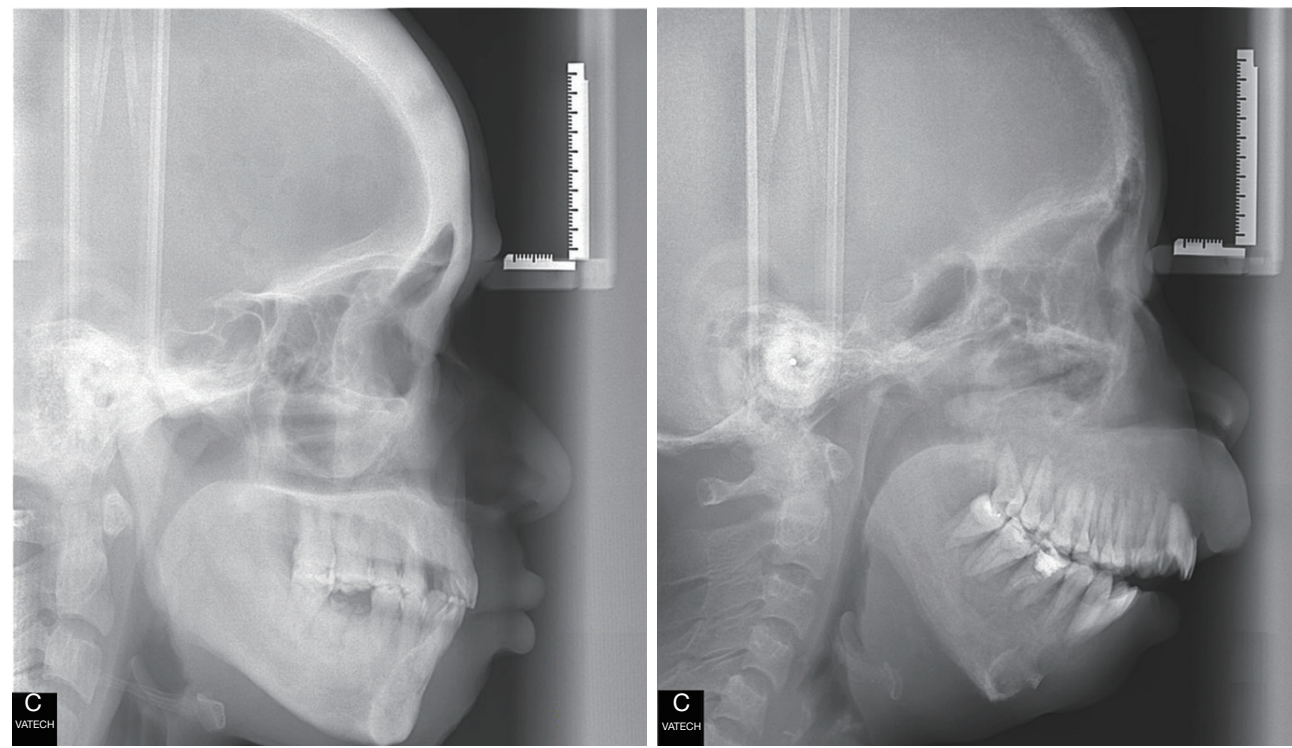

Figure 1 Cephalometric X-rays of a patient with secondary hyperparathyroidism (SHPT) and a patient with Sagliker syndrome, respectively.

Table 1 Participant demographics

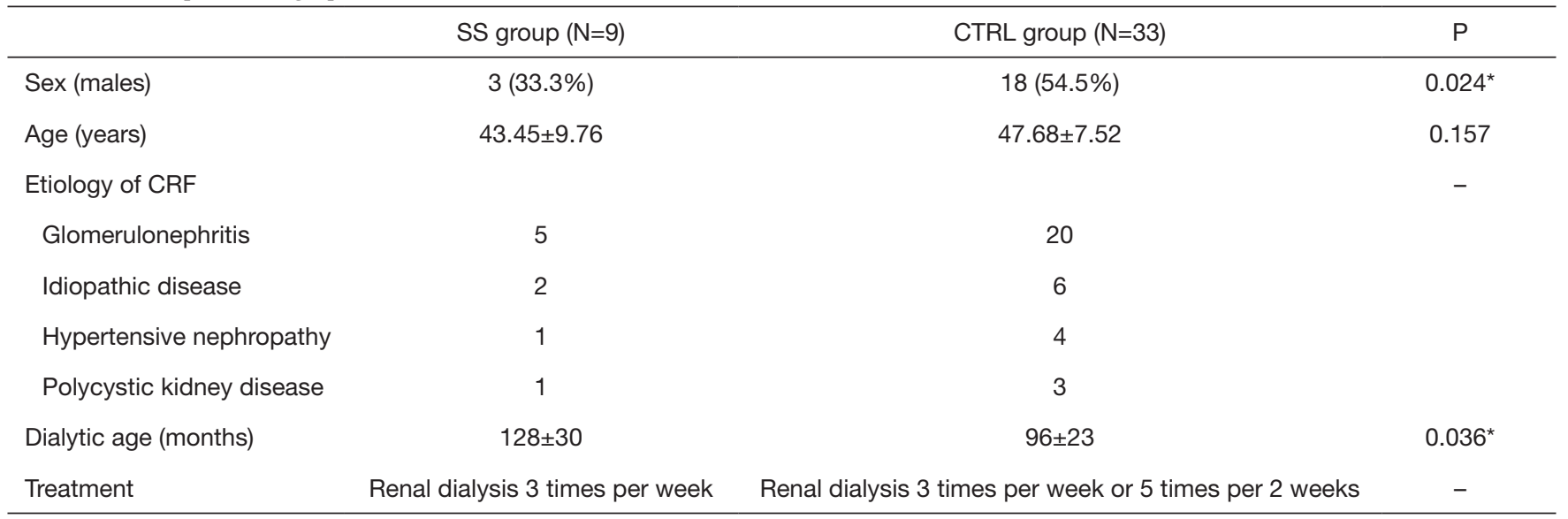

*, significance level, $\mathrm{P}<0.05$. SS, Sagliker syndrome; CTRL, control; CRF, chronic renal failure.

measured using standard automated techniques. A standard radioimmunoassay kit (Cobas e411, Roche Diagnostics, USA) was used to estimate intact parathyroid hormone (iPTH) (ng/L).

\section{Statistical analyses}

Cephalometric variables were calculated as the mean and standard deviation. Student's $t$ test or Mann-Whitney $\mathrm{U}$ tests were used to compare variables or a non-normal distribution $\mathrm{P}<0.05$ using All analyses were performed in
SPSS Statistics 22 (Chicago, IL, USA).

\section{Results}

\section{Baseline demographics}

We enrolled 9 patients with SS and 33 patients with simple SHPT and evaluated their cephalometric craniofacial features. The SS group comprised 3 males and 6 females (ratio, $0.5: 1)$ with an average age $( \pm \mathrm{SD})$ of $43.45 \pm 9.76$ years and a dialytic age $( \pm$ SD) of $128 \pm 30$ months. The SHPT 


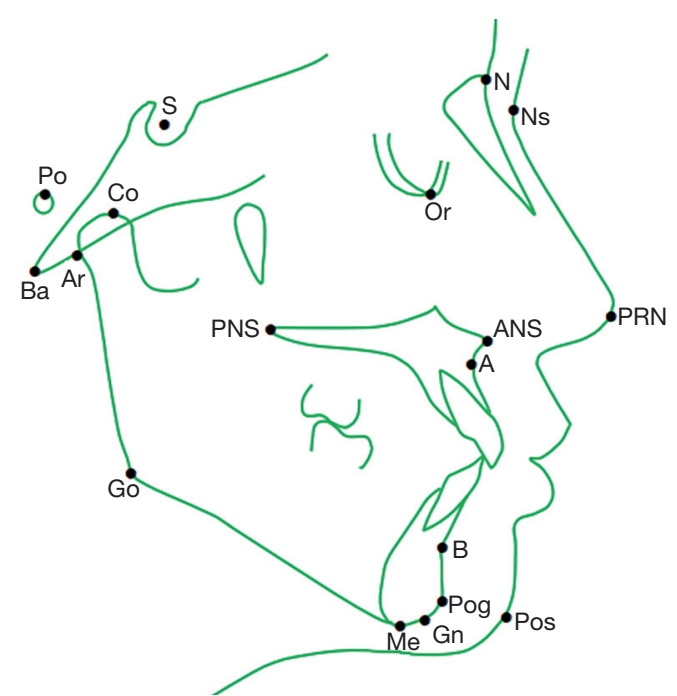

Figure 2 Cephalometric landmark points: subspinale (A); supramentale $(\mathrm{B})$; nasion $(\mathrm{N})$; nasion of soft tissue $(\mathrm{Ns})$; orbitale (Or); pronasale (PRN); anterior nasal spine (ANS); articulare (Ar); basion (Ba); condylion (Co); glabella (G); gnathion (Gn); gonion (Go); menton (Me); anterior nasal spine (ANS); porion (Po); posterior nasal spine (PNS); pogonion of soft tissue (Pos); pogonion (Pog); sella (S).

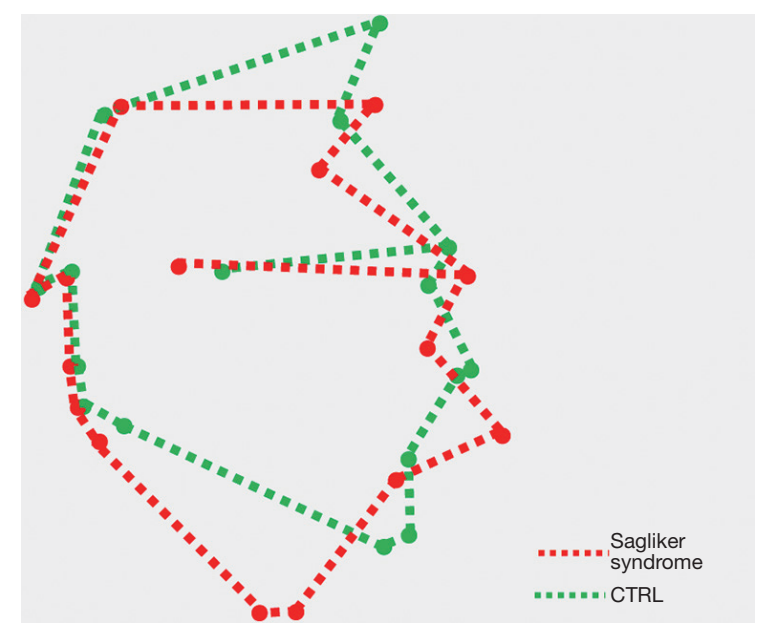

Figure 3 Comparative illustration of the morphological craniofacial bone anomalies of patients in the Sagliker syndrome (red lines). and control groups (green lines).

group comprised 18 males and 15 females (ratio, 1.2:1), who had an average age $( \pm \mathrm{SD})$ of $47.68 \pm 7.52$ years and a dialytic age $( \pm \mathrm{SD})$ of $96 \pm 23$ months. The demographic data of all participants are listed in Table 1. Statistically significant differences were observed between the groups with respect to sex $(\mathrm{P}=0.024)$, age $(\mathrm{P}=0.157)$, and dialytic age $(\mathrm{P}=0.036)$.

\section{Cephalometric measurements}

Inter- and intra-reliability tests revealed no significant differences in the digitized cephalometric variables, indicating all radiographic parameters to have satisfactory reproducibility and the analyses to be reliable. To study the cephalometric craniofacial features of the patients with SS, we measured their cephalometric linear and angular parameters for comparison with those of the control group. A descriptive and comparative analysis of all cephalometric variables is presented in Table 2.

The lower anterior facial height (ANS-ME) and total anterior facial height $(\mathrm{N}-\mathrm{Me})$ measurements of patients with SS were significantly increased compared to those of the controls $(\mathrm{P}<0.05)$. The mandibular body length $(\mathrm{Go}-\mathrm{Me})$ of the SS group was statistically significantly shorter than that of the controls $(\mathrm{P}<0.05)$. In terms of angular measurements, the angles between the Frankfort horizontal, palatal, and occlusal planes and the mandibular plane in participants with SS were greater than those in the control group. The gonial angle (Ar-Go-Me) also showed a statistically significant increase in the SS group compared to the controls $(\mathrm{P}<0.05)$.

In regard to anteroposterior skeletal measurements, compared to the controls. In regard to anteroposterior skeletal measurements, compared to the controls, patients with SS exhibited a significantly larger maxillary protrusion angle, and the relative position of the maxilla to the mandible was ANB. Meanwhile, the horizontal position of the mandible (SNB) and facial angle (NP-FH) showed a decrease compared to the controls, although no statistically significant differences were observed. The orofacial bones of patients with SS also exhibited an enlarged gonion angle (Ar-Go-Me) and S-Ar-Go, without any mandibular prognathism (S-N-PG), and no significant differences were found in the maxillary inclination angle (NL/NSL), anterior cranial base (S-N), depth of the bony pharyngeal space (BAPNS), or mandibular ramus length (CD-GO).

In patients with SS, soft-tissue findings included elongation of the distance from the upper lip to Ricketts' E-line length (UL-EP), an increased upper lip inclination angle (AsUL-FH), and some reduction in the lower lip inclination angle (BsLL-FH) and soft-tissue facial angle (NsPgs/FH) compared to the control group. However, no significant differences were found in the chin thickness, nose length (N-PRN), nasolabial angle (NLA), nasal tip protrusion (S-N-PRN), or distance from the lower 
Table 2 Comparative analysis of skeletal cephalometric measurements between the SS group and the control group

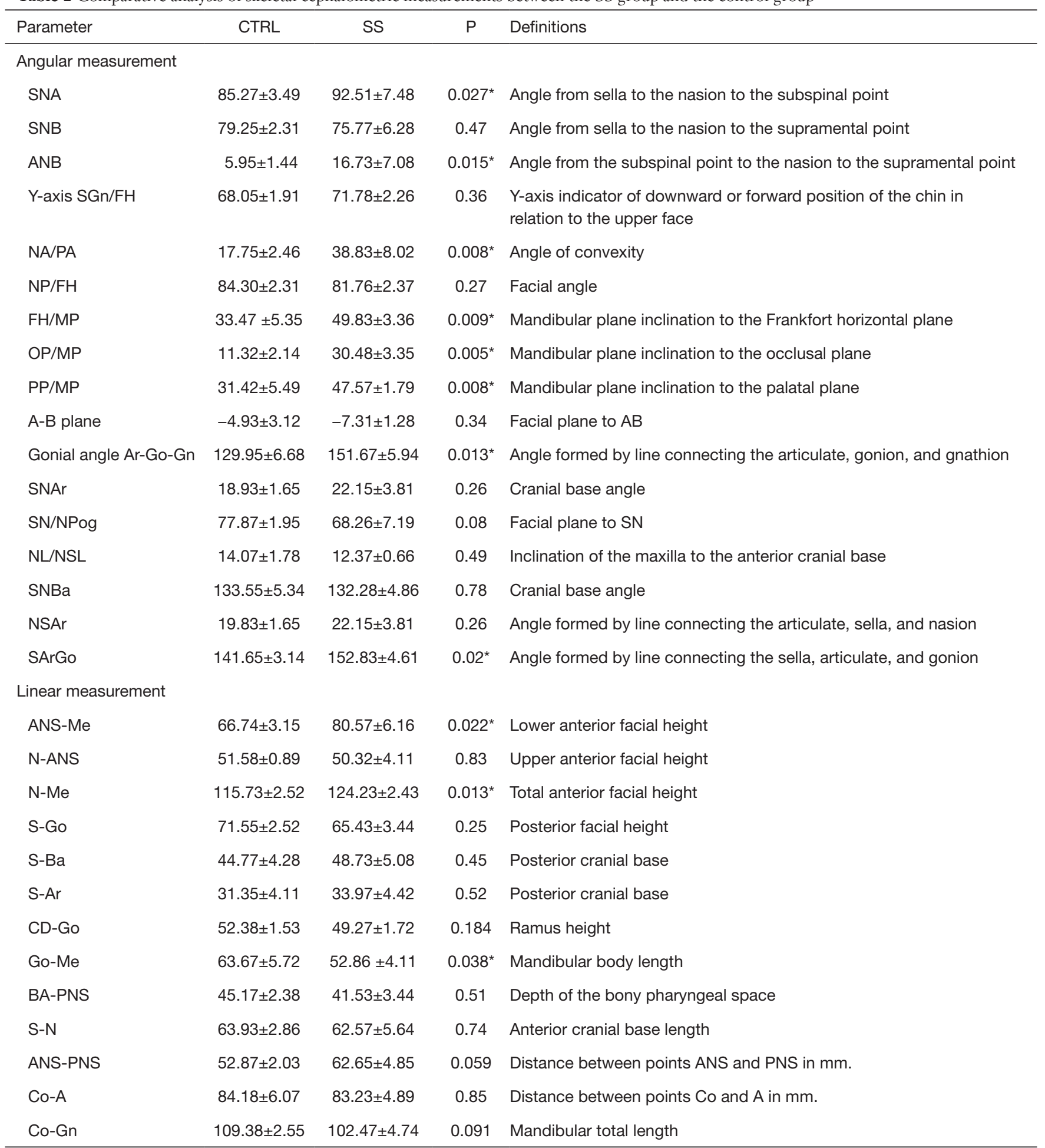

Table 2 (continued) 
Table 2 (continued)

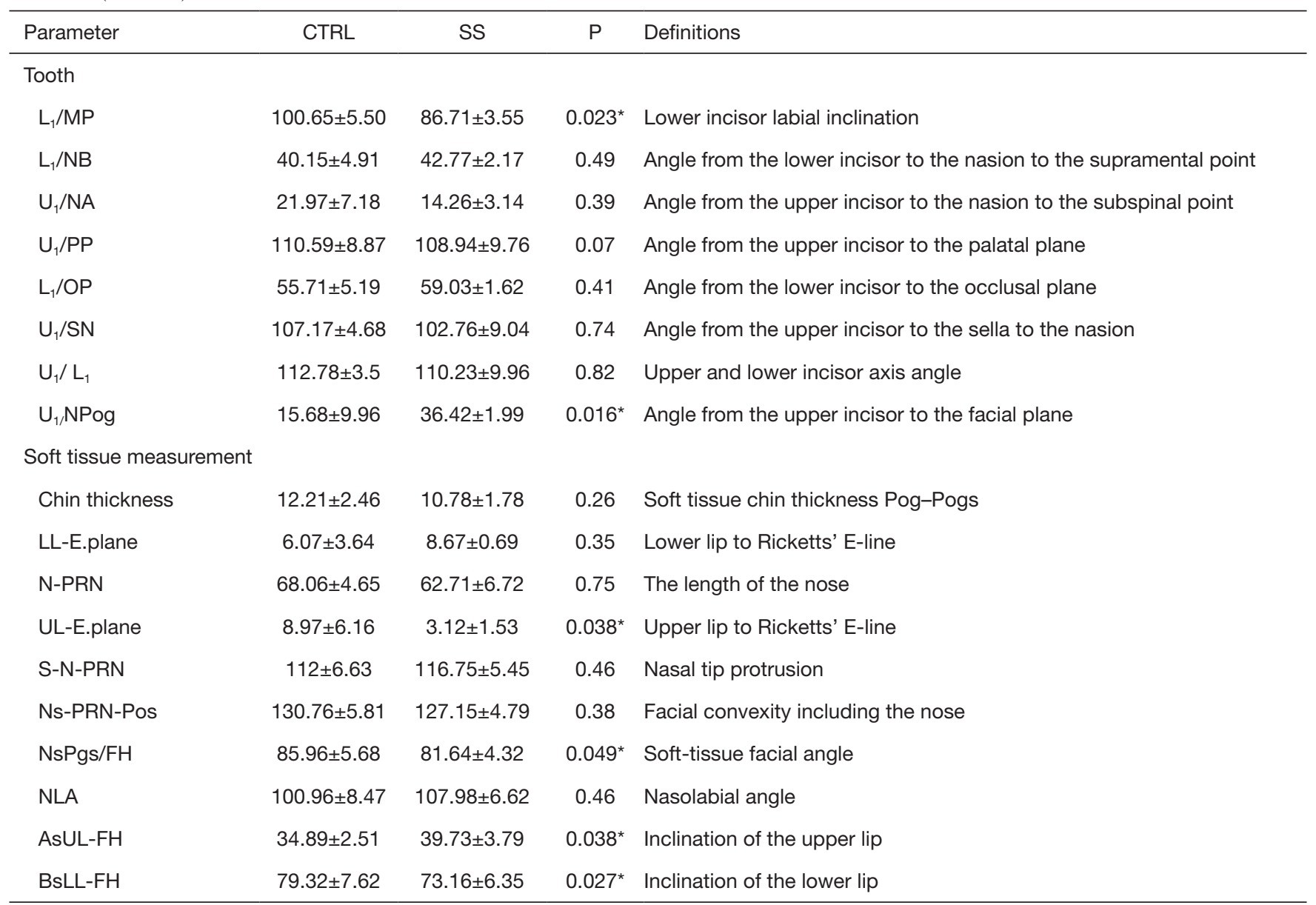

*, significance level, $\mathrm{P}<0.05$. SS, Sagliker syndrome; CTRL, control.

lip to Ricketts' E-line (LL-EP) between the groups $(\mathrm{P}>0.05)$.

Regarding dental features, the $\mathrm{U}_{1} / \mathrm{NPog}$ was significantly larger in the SS group than in the control group, while the lower incisor labial inclination angle $\left(\mathrm{L}_{1} / \mathrm{MP}\right)$ was significantly decreased. The $\mathrm{U}_{1} / \mathrm{NA}, \mathrm{U}_{1} / \mathrm{PP}, \mathrm{U}_{1} / \mathrm{SN}$, and $\mathrm{U}_{1} / \mathrm{L} 1$ of the SS patients showed reductions compared to those of the controls; however, no statistical significance was found. Furthermore, among the participants with SS, there were $6(66.7 \%)$ cases of class II skeletal malocclusion, $2(22.2 \%)$ cases of class III malocclusion, and $1(11.1 \%)$ case of normal occlusion, whereas most of the controls exhibited normal occlusion.

\section{Serum biochemical parameters and iPTH levels}

As shown in Figure 4, the serum levels of iPTH, as well as those of ALP, were significantly higher in the SS group than in the control group $(\mathrm{P}<0.001)$; however, the levels of serum calcium and phosphorus did not differ significantly between the groups. Next, we further determined the relationship between serum calcium, iPTH, and ALP. As shown in Figure 5, in the control group, when $\left(\mathrm{Ca}^{2+}\right)$ was $2.0-3.0 \mathrm{mmol} / \mathrm{L}$, the levels of serum iPTH increased; however, the iPTH levels were decreased in higher $\mathrm{Ca}^{2+}$ concentrations. However, trends about the $\mathrm{Ca}^{2+}$ and serum iPTH were not observed for serum iPTH in the SS group. Furthermore, the levels of serum iPTH were positively correlated with the ALP levels in both the SS group $(\mathrm{Y}=0.8467 \mathrm{X}-874.4, \mathrm{R}=0.778, \mathrm{P}<0.05)$ and the control group $(\mathrm{Y}=3.530 \mathrm{X}-261.9, \mathrm{R}=0.621, \mathrm{P}<0.001)$.

\section{Discussion}

A Turkish population-based study of 5,000 patients 

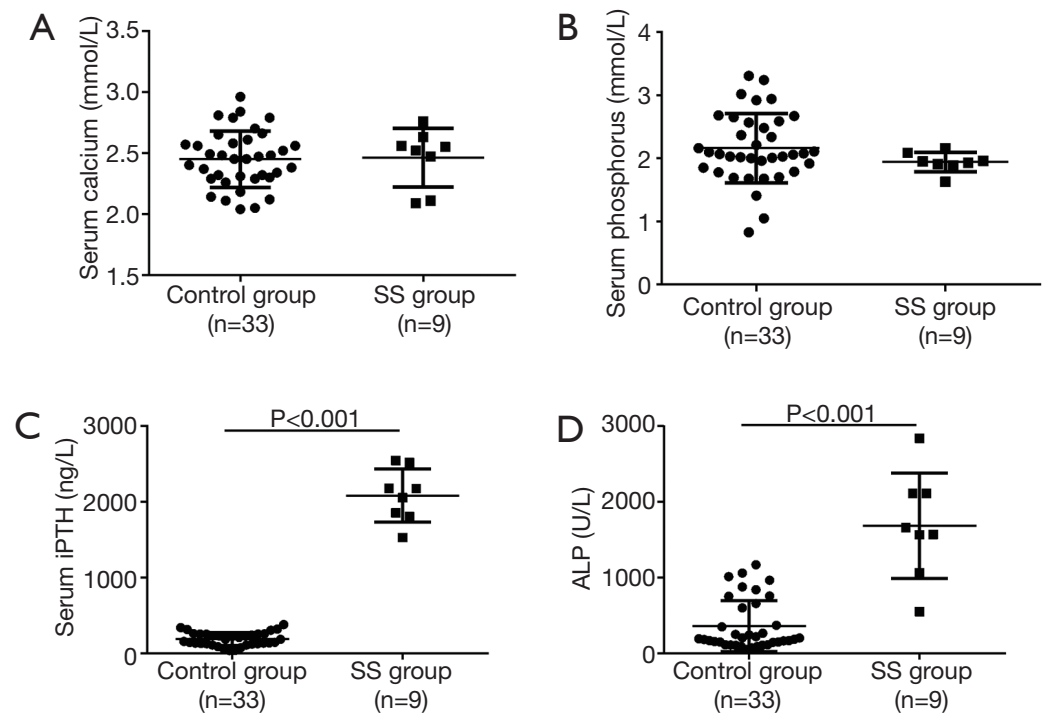

Figure 4 Biochemical characteristics of patients in Sagliker syndrome (SS) and control (CTRL) groups. (A) Levels of serum calcium; (B) levels of serum phosphorus; (C) levels of serum intact parathyroid hormone (iPTH); (D) levels of serum alkaline phosphatase (ALP).
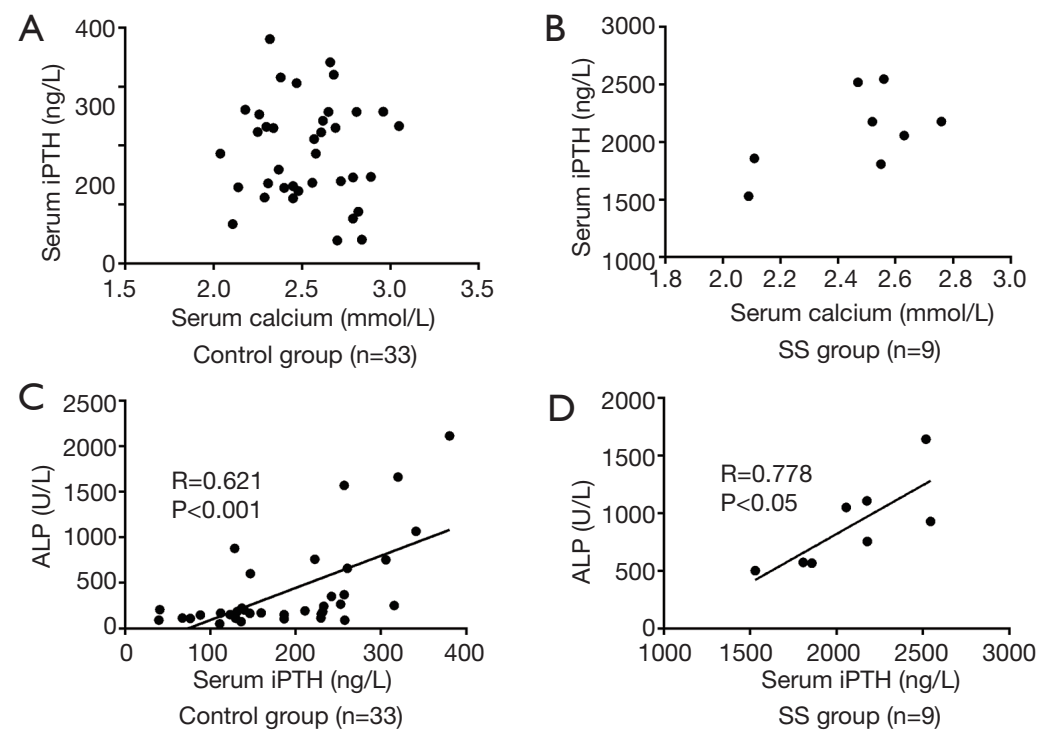

Figure 5 Relationships between serum calcium, intact parathyroid hormone (iPTH), and alkaline phosphatase (ALP) in the Sagliker syndrome (SS) and control groups. (A) Relationships between serum calcium and iPTH in control groups; (B) relationships between serum calcium and iPTH in SS groups; (C) relationships between serum iPTH and ALP in control groups; (D) relationships between serum iPTH and ALP in SS groups.

receiving hemodialysis reported SS to have an approximate incidence of $0.5 \%$ (6). SS causes tremendous changes and complications to occur throughout the body, particularly in the skull bones and maxillofacial skeleton, which have irreversible and devastating effects on the individual's appearance $(3,7)$, as well as their psychological health (8).
It also entails numerous SHPT signs and symptoms which affect multiple systems in the body (9), and poses a severe threat to patients' lives and life quality (10). Therefore, it is necessary to uncover the relationship between biochemical variables and the craniofacial features of patients with SS, so as to further provide a theoretical basis for the early 
diagnosis and clinical prevention of this syndrome.

The causes of abnormal growth of the craniofacial region have been a focus of interest in orthodontic and dentofacial orthopedic research for some time. However, due to the rare incidence of SS, the specific skeletal patterns of patients affected by this condition have not yet been fully elucidated. In our lateral cephalometric study we observed that the majority of linear parameters, as indicators of craniofacial growth (11), were increased in the SS group in comparison to the control group. Although our study was based on a relatively small sample size, as far as we know, it is the first evaluation of the craniofacial morphological features of individuals with SS.

Analysis of the cephalometric angular measurements of SS patients and controls revealed that, in terms of anteroposterior craniofacial dimensions, the SNA, ANB, and NA/PA showed a significant increase in the SS group, while the SNB and NP/FH did not differ significantly between the groups. Also, significant maxillary protrusion and slight but not significant increases were found in mandibular changes of patients with SS. As a critical evaluation of the sagittal position of the maxilla to the mandible, the ANB angle showed a statistically significant increase in SS patients, which is well in keeping with observations in other studies. Among these researches, Uzel's lateral cephalometric analysis observed severe skeletal class II malocclusion with mandibular deficiency in the majority of participants with SS (12). Vertical skeletal measurements showed that patients with SS have statistically significantly increased lower and total anterior facial heights, whereas the upper anterior and total posterior facial heights did not show significant differences between the two groups. We also found that patients with SS exhibited limited cranial skeletal alterations, with the anterior maxilla and mandible being the most affected parts of the viscerocranium, and no statistically significant changes being observed in the posterior maxilla and mandible, or other cranial skeletal parts. The Ar-Go-Me, S-Ar-Go, and the angles between the Frankfort horizontal (FH), palatal (PP), and occlusal planes $(\mathrm{OP})$ and the mandibular plane (MP) were increased in the SS patients compared with the controls. Our results revealed that the mandible did not show significant changes in its sagittal direction, rotating backwards and vertically downwards. Overall, the facial features of patients with SS are characterized by a convex profile with excessive vertical growth of the maxilla (vertical maxillary excess) and mandibular retrusion; this is consistent with observations made in a previous study of 4 SS patients (12), and the very strong impression of "lion face" observed in patients.

On cephalometric X-ray, computed tomography, or MRI, we observed very unique and distinctive irregularities and changes in bone density in the patients with SS, as well as striking maxillary excess and mandibular deficiencies, and nasal bone destruction (Figure 6 and Figure 1). The 9 SS patients exhibited teeth with irregular shapes and locations (Figure 6 and Figure 1); in 4 cases, soft, large, tumor-like growths were observed, especially in the upper oral cavity (Figure 6H,I), and some patients exhibited multiple ectopic calcifications (Figure 6D,E,F), scapula deformity (Figure 6F), and long bone deformity (Figure 6C). Some clinical trials have demonstrated that parathyroidectomy and total parathyroidectomy with autotransplantation (TPTX + AT) can both improve a range of clinical SS symptoms $(13,14)$, which not only aids in preventing the development of facial and jaw deformity and height retraction, but also improves the psychiatric manifestations of SS, such as depression and despair (15). Our medical record review also confirmed that TPTX+ AT can significantly reduce the size of tumor-like tissue (data not shown) and serum biochemical parameters (16) of patients in the short term. However, long-term retrospective and clinical follow-up study is needed to determine whether it can attain improvements in terms of patients' quality of life and prognosis in the long term.

A study by China-Japan Friendship Hospital showed that sex, age, duration of dialysis, and high serum levels of ALP are potential risk factors for SS (3); evidence from our study is consistent with these findings. Our study involved significantly more women with SS than men, at a ratio of 2:1, suggesting that estrogen may play a role in the pathophysiology of SS. In another study by Chertow et al. (17), after clinical trials, a direct relationship was reported between dialysis vintage and the severity of hyperparathyroidism. As the dialytic duration increases, there is a gradual decline and loss of residual renal function, which results in aggravation of phosphorus retention and stimulates hypertrophy of the parathyroid glands, gradually forming nodular or high-functioning adenomatoid hyperplasia (18). In a previous study of individuals with SS before hemodialysis initiation, the average serum calcium and phosphate levels were 6-7 and 7-8 mg/dL, respectively; in addition, an increase was observed in ALP levels (120 $240 \mathrm{U} / \mathrm{L})$, while the iPTH levels were $\geq 3.5$ times the normal level (180-240 pg/mL) (1). In our study, we found that the serum calcium and phosphate levels of patients with SS were not especially high, while much higher levels 

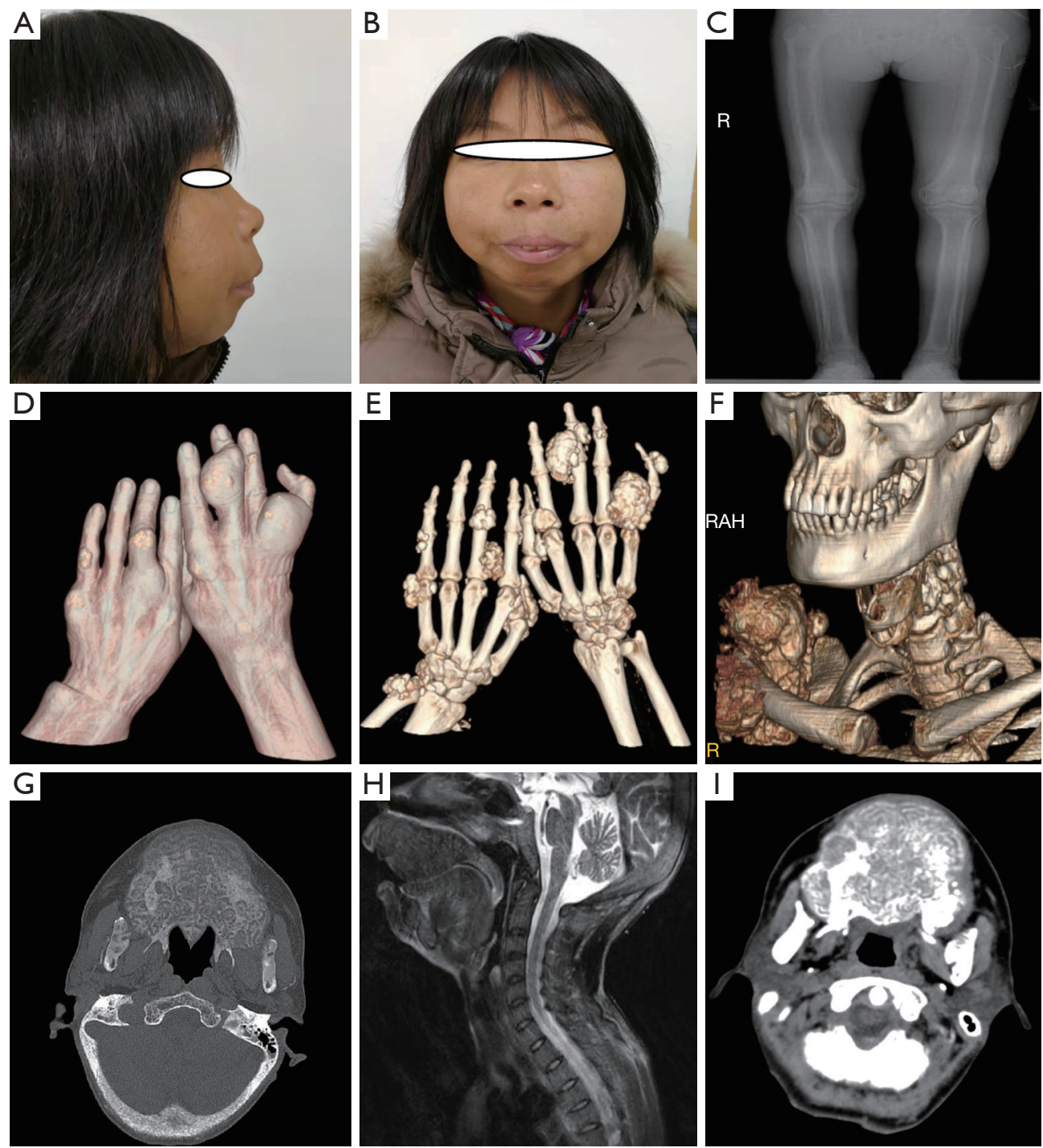

Figure 6 Signs of patient with hyperparathyroidism. (A,B) Maxillary and mandibular bone changes, and dental malocclusion; (C) long bone deformities; (D,E,F) multiple ectopic calcifications; (G) skull bone tomography showing salt-and-pepper syndrome in a patient with SS; (H,I) tumor-like tissue growths in the upper oral cavity under computed tomography and MRI.

of ALP and iPTH were detected. One of the reasons for this difference may be related to the fact that some bias may have existed due to the small sample size. However, a study by Hoenderop et al. (19) demonstrated that, when $\left(\mathrm{Ca}^{2+}\right)$ is $1.88-2.63 \mathrm{mmol} / \mathrm{L}$, a low serum calcium level stimulates the secretion of PTH; conversely, a high serum calcium level inhibits its secretion via a closed-loop feedback mechanism. The results of our control group are in agreement with these observations; meanwhile, hypercalcemia was not associated with inhibited iPTH secretion in the SS group. It is tempting to consider that there may be a mechanism for preserving ultra-high concentrations of iPTH and ALP, even though high serum calcium levels in patients with SS result in the clinical manifestations of "lion face", future studies may clarify this possibility.

In recent years, researchers have paid more attention to SS in an effort to understand the mechanisms behind the development in SS in patients with SHPT and to determine the best treatment for SS. The reasons for SS developing in certain patients with SS are still unclear. A study of SS patients indicated that missense mutations of the guanine nucleotide-binding protein G(s) 1 (GNAS1) gene could be 
implicated in the genesis of SS rather than abnormalities in chromosomes or the calcium-sensing receptor gene (4). Moreover, Demirhan also conducted screening for mutations in all the 13 exons of GNAS1 gene, all 3 exons of FGF23, and all 18 exons in FGFR3 genes in 23 patients. They found that 17 genetic abnormalities in GNAS1 were detected in $73.9 \%$ (17 of 23) patients (20). Due to financial limitations of the current study, we were unable to carry out molecular or genetic investigations. The major limitations of this study are the small number of participants. Future large-scale research should be carried out, and patients should be followed up with cephalometric and biochemical evaluations to gain a more accurate understanding of craniofacial changes in SS, so as to further the theoretical basis for the early diagnosis and prevention of this syndrome.

\section{Conclusions}

Patients with SS exhibit facial and biochemical anomalies compared to patients with SHPT. Female sex, duration of dialysis, and high serum levels of iPTH and ALP may be potential risk factors for SS.

\section{Acknowledgments}

Funding: This study was supported by Zhuhai Scientific and Technological Project Fund (No. 20161027F060002 and No. 20181117E030007). Guangdong Administration of Traditional Chinese Medicine Project Fund (No. 20191072), and Guangdong Medical Scientific and Technological Project Fund (No. A2020364)

\section{Footnote}

Reporting Checklist: The authors have completed the STROBE reporting checklist. Available at https://dx.doi. org/10.21037/atm-21-1544

Data Sharing Statement: Available at https://dx.doi. org/10.21037/atm-21-1544

Conflicts of Interest: All authors have completed the ICMJE uniform disclosure form (available at https://dx.doi. org/10.21037/atm-21-1544). The authors have no conflicts of interest to declare.

Ethical Statement: The authors are accountable for all aspects of the work in ensuring that questions related to the accuracy or integrity of any part of the work are appropriately investigated and resolved. The study was approved by the Ethics Committee of the fifth affiliated hospital of Sun Yat-sen University. Participants gave their consent after being fully informed of the study's purpose. The study was conducted in accordance with the Declaration of Helsinki (as revised in 2013)

Open Access Statement: This is an Open Access article distributed in accordance with the Creative Commons Attribution-NonCommercial-NoDerivs 4.0 International License (CC BY-NC-ND 4.0), which permits the noncommercial replication and distribution of the article with the strict proviso that no changes or edits are made and the original work is properly cited (including links to both the formal publication through the relevant DOI and the license). See: https://creativecommons.org/licenses/by-nc-nd/4.0/.

\section{References}

1. Sagliker Y, Balal M, Sagliker Ozkaynak P, et al. Sagliker syndrome: Uglifying human face appearance in late and severe secondary hyperparathyroidism in chronic renal failure. Semin Nephrol 2004;24:449-55.

2. Sagliker $Y$, Acharya V, Ling Z, et al. International Study on Sagliker Syndrome and Uglifying Human Face Appearence in Severe and Late Secondary Hyperparathyroidism in Chronic Kidney Disease Patients. J Ren Nutr 2008;18:114-7.

3. Chen XH, Shen B, Zou JZ, et al. Clinical status of Sagliker syndrome: a case report and literature review. Ren Fail 2014;36:800-3.

4. Mejía Pineda A, Aguilera ML, Meléndez HJ, et al. Sagliker syndrome in patients with secondary hyperparathyroidism and chronic renal failure: Case report. Int J Surg Case Rep 2015;8C:127-30.

5. Mohebi-Nejad A, Gatmiri SM, Abooturabi SM, et al. Diagnosis and treatment of Sagliker syndrome: a case series from Iran. Iran J Kidney Dis 2014;8:76-80.

6. Yildiz I, Sagliker Y, Demirhan O, et al. International Evaluation of Unrecognizably Uglifying Human Faces in Late and Severe Secondary Hyperparathyroidism in Chronic Kidney Disease. Sagliker Syndrome. A Unique Catastrophic Entity, Cytogenetic Studies for Chromosomal Abnormalities, Calcium-Sensing Receptor Gene and GNAS1 Mutations. Striking and Promising Missense Mutations on the GNAS1 Gene Exons 1, 4, 10, 4. 
J Ren Nutr 2012;22:157-61.

7. Sagliker Y, Acharya V, Golea O, et al. Is survival enough for quality of life in Sagliker Syndrome-uglifying human face appearances in chronic kidney disease? J Nephrol 2008; 21 Suppl 13:S134-8.

8. Ozenli Y, Giray S, Sagliker Y, et al. A Controlled Study of Psychiatric Manifestations and Electroencephalography Findings in Chronic Kidney Disease Patients With Sagliker Syndrome. J Ren Nutr 2010;20:S51-5.

9. Goodman WG, Goldin J, Kuizon BD, et al. Coroaryartery calcification in young dults with end-stage renal disease who are undergoing dialysis. $\mathrm{N}$ Engl J Med 2000;342:1478-83.

10. Fraser WD. Hyperparathyroidism. Lancet 2009;374:145-58.

11. Al-Thomali Y, El-Bialy TH. Cephalometric craniofacial features of growing patients with chronic renal failure. Arch Oral Biol 2012;57:257-63.

12. Uzel A, Uzel I, Sagliker Y, et al. Cephalometric Evaluation of Patients With Sagliker Syndrome: Uglifying Human Face Appearance in Severe and Late Secondary Hyperparathyroidism in Chronic Renal Failure Patients. J Ren Nutr 2006;16:229-32.

13. Cheng SP, Lee JJ, Liu TP, et al. Parathyroidectomy improves symptomatology and quality of life in patients with secondary hyperparathyroidism. Surgery 2014;155:320-8.

Cite this article as: Mi JP, He P, Shi K, Feng SY, Chen XZ, He QQ, Zhao MY, Ge PJ, Fan YP. Cephalometric craniofacial features of patients with Sagliker syndrome: a primary analysis of our experience. Ann Transl Med 2021;9(12):963. doi: 10.21037/atm-21-1544
14. Lau WL, Obi Y, Kalantar-Zadeh K. Parathyroidectomy in the Management of Secondary Hyperparathyroidism. Clinical Journal of the American Society of Nephrology Cjasn 2018;13:952-61.

15. Zhang L, Yao L, Bian WJ, et al. Severe uremic leontiasis ossea ameliorated by total parathyroidectomy. Kidney International 2009;76:1118.

16. Mi JP, Liao ZP, Pei XF, et al. Postsurgical Evaluation of Secondary Nephrogenic Hyperparathyroidism. Current Medical Science 2019;39:259-64.

17. Chertow GM, Plone M, Dillon MA, et al. Hyperparathyroidism and dialysis vintage. Clinical Nephrology 2000;54:295-300.

18. Wang HY, Liu GL, Wang J, et al. Analysis of risk factors related to patients with Sagliker syndrome. Chin J Nephrol 2015;31:749-754.

19. Hoenderop JG, Nilius B, Bindels RJ. Molecular mechanism of active $\mathrm{Ca} 2+$ reabsorption in the distal nephron. Annu Rev Physiol 2002;64:529-49.

20. Demirhan O, Arslan A, Sagliker Y, et al. Gene Mutations in Chronic Kidney Disease Patients With Secondary Hyperparathyroidism and Sagliker Syndrome. Journal of Renal Nutrition 2015;25:176-186.

(English Language Editor: J. Reynolds) 